9 Page T, Bakay B, Nissinen E, Nyhan W L. Hypoxanthine-guanine phosphoribosyltransferase variants: correlations of clinical phenotype with enzyme activity. Journal of Inherited Metabolic Diseases 1981; 4: 203-7.
10 Cameron J S, Simmonds H A, Webster D R, Wass V, Sahota A. Problems of diagnosis and in vitro enzyme instability in an adolescent with hypoxanthineguanine phosphoribosyltransferase deficiency presenting in acute renal fail- ure. J Clin Chem Clin Biochem 1982; 20: 353 .

\title{
Lean, dry gout patients
}

\author{
L. GAIL DARLINGTON \\ From the Epsom District Hospital, Epsom, Surrey
}

Hypertriglyceridaemia is common in gout. ${ }^{2}$ Obesity may increase triglyceride concentrations, as may alcohol, ${ }^{3}$ but it remains uncertain whether obesity and alcohol, alone or in combination, are sufficient to explain the hyperlipidaemia in all cases. We looked for abnormal lipid concentrations in non-obese patients with gout who drank little or no alcohol to determine whether the hy perprebetalipoprote in a $\mathrm{mia}$ associated with gout occurred in such a lean and abstemious group.

All patients were of desirable weight or less for their age and frame ${ }^{4}$ and no patient drank more than one pint of beer per day or its equivalent. Such patients are rare, and only seven were found in four years from a busy clinic.

Fasting concentrations of lipid and lipoprotein were measured in serum at a laboratory with its own control population.' Serum uric acid concentrations were determined for the patients with gout but unfortunately data for the control population were not available.

Readings for serum cholesterol, triglyceride, $\beta$-lipoprotein and prebeta lipoprotein concentrations in the patients with gout lay within 2 standard deviations of the corresponding mean for controls. This means that they were within the $95 \%$ confidence limits for the control population and it is therefore unlikely that there is any real difference between the patients with gout and control populations.

In spite of the small numbers of these, 'lean, dry' patients, the results revealed no intrinsic hyperlipidaemia in subjects with gout when obesity and an excess of alcohol were removed as causes of hypertriglyceridaemia.

\section{References}

1 Darlington L G, Scott J T. Plasma lipid levels in gout. Ann Rheum Dis 1972; 31: 487-9.

2 Mielants $H$, Veys E M, de Weerdt A. Gout and its relations to lipid metabolism. I. Serum uric acid, lipid and lipoprotein levels in gout. Ann Rheum Dis 1973; 32: 501-5.

3 Gibson T, Grahame R. Gout and hyperlipidaemia. Ann Rheum Dis 1974; 33: $298-303$.

4 Metropolitan Life Insurance Company. Build and blood pressure study. Chicago: Society of Actuaries, Ill. 1959.

5 Slack J, Noble N, Meade T W, North W $R$ S. Lipid and lipoprotein concentrations in 1,604 men and women in working populations in north-west London. Br Med J 1977; ii: 353-6.

\section{Cardiovascular disease and gout: a function of sex and age?}

\author{
D. G. MACFA RLANE
}

From the University Department of Medicine, Bristol Royal Infirmary, Bristol

Hypertriglyceridaemia is common in patients with gout and hyperuricaemia but it is still not known whether this results from a link between purine and lipoprotein metabolism or whether they occur together due to other associated facts, particularly obesity and high alcohol intake, both of which are commonly found in patients with hyperuricaemia. ${ }^{1}$ Nor is it firmly established whether patients with gout are, in fact, predisposed to premature cardiovascular disease, and, if so, whether the raised serum uric acid concentration operates as an independent risk factor, or only via its association with hypertriglyceridaemia and hypertension, which are established as cardiovascular risk factors in their own right. ${ }^{2}$ Another possibility-namely, that a raised serum uric acid concentration causes platelet hyperaggregatability and hence thrombosis - has recently been investigated in our unit with negative results. $^{3}$

Over a period of two and half years 